\title{
The Ramazzini Institute 13-week pilot study on glyphosate and Roundup administered at human-equivalent dose to Sprague Dawley rats: effects on the microbiome
}

Qixing Mao ${ }^{1,2 \dagger}$, Fabiana Manservisi ${ }^{3,4 \dagger}$, Simona Panzacchi ${ }^{3}$, Daniele Mandrioli, ${ }^{3,5}$, llaria Menghetti ${ }^{3}$, Andrea Vornoli ${ }^{3}$, Luciano Bua ${ }^{3}$, Laura Falcioni ${ }^{3}$, Corina Lesseur ${ }^{6}$, Jia Chen ${ }^{6}$, Fiorella Belpoggi ${ }^{3^{*}}$ and Jianzhong $\mathrm{Hu}^{1}$

\begin{abstract}
Background: Glyphosate-based herbicides (GBHs) are broad-spectrum herbicides that act on the shikimate pathway in bacteria, fungi, and plants. The possible effects of GBHs on human health are the subject of an intense public debate for both its potential carcinogenic and non-carcinogenic effects, including its effects on microbiome. The present pilot study examines whether exposure to GBHs at doses of glyphosate considered to be "safe" (the US Acceptable Daily Intake - ADI - of $1.75 \mathrm{mg} / \mathrm{kg}$ bw/day), starting from in utero, may modify the composition of gut microbiome in Sprague Dawley (SD) rats.

Methods: Glyphosate alone and Roundup, a commercial brand of $\mathrm{GBHs}$, were administered in drinking water at doses comparable to the US glyphosate ADI $(1.75 \mathrm{mg} / \mathrm{kg}$ bw/day) to F0 dams starting from the gestational day (GD) 6 up to postnatal day (PND) 125. Animal feces were collected at multiple time points from both F0 dams and F1 pups. The gut microbiota of 433 fecal samples were profiled at V3-V4 region of $16 \mathrm{~S}$ ribosomal RNA gene and further taxonomically assigned and assessed for diversity analysis. We tested the effect of exposure on overall microbiome diversity using PERMANOVA and on individual taxa by LEfSe analysis.
\end{abstract}

Results: Microbiome profiling revealed that low-dose exposure to Roundup and glyphosate resulted in significant and distinctive changes in overall bacterial composition in F1 pups only. Specifically, at PND31, corresponding to pre-pubertal age in humans, relative abundance for Bacteriodetes (Prevotella) was increased while the Firmicutes (Lactobacillus) was reduced in both Roundup and glyphosate exposed F1 pups compared to controls.

Conclusions: This study provides initial evidence that exposures to commonly used GBHs, at doses considered safe, are capable of modifying the gut microbiota in early development, particularly before the onset of puberty. These findings warrant future studies on potential health effects of GBHs in early development such as childhood.

Keywords: Roundup, Glyphosate, Gut microbiome, Early developmental stage

\footnotetext{
* Correspondence: belpoggif@ramazzini.it; http://www.ramazzini.org

${ }^{\dagger}$ Equal contributors

${ }^{3}$ Cesare Maltoni Cancer Research Center (CMCRC), Ramazzini Institute (RI), Via

Saliceto, 3, 40010 Bentivoglio, Bologna, Italy

Full list of author information is available at the end of the article
}

(c) The Author(s). 2018 Open Access This article is distributed under the terms of the Creative Commons Attribution 4.0 International License (http://creativecommons.org/licenses/by/4.0/), which permits unrestricted use, distribution, and reproduction in any medium, provided you give appropriate credit to the original author(s) and the source, provide a link to the Creative Commons license, and indicate if changes were made. The Creative Commons Public Domain Dedication waiver (http://creativecommons.org/publicdomain/zero/1.0/) applies to the data made available in this article, unless otherwise stated. 


\section{Background}

Glyphosate (IUPAC chemical name N-(phosphonomethyl) glycine) is the active ingredient of the most widely applied herbicide worldwide, glyphosate-based herbicides (GBHs), including the best-known formulation Roundup. The substance glyphosate was initially discovered in 1950 by a Swiss chemist, Henri Martin, at the pharmaceutical company Cilag [1]. Its herbicidal properties were not discovered for another 20 years. Since glyphosate was patented in 1974 by Monsanto as a herbicide, approximately 9.4 million tons of GBHs have been sprayed, nearly half a pound of glyphosate on every cultivated acre of land globally [2]. Furthermore, after the introduction of genetically modified (GM) crops that are glyphosate-tolerant in 1996, usage of GBHs has skyrocketed; about two-thirds of the total GBHs usage took place in recent decades. According to the National Academy of Sciences report [3], in 2014 alone, annual glyphosate usage in agriculture industry exceeded 110 million kilograms. Besides GM crops, farmers also apply GBHs on non-GM crops in order to accelerate the harvest. This practice, also known as desiccation, has led to significant dietary exposure to the residues of glyphosate and its primary metabolite AMPA (aminomethylphosphonic acid) $[4,5]$.

The primary herbicidal function of glyphosate is to inhibit a key plant enzyme, namely 5-enolpyruvylshikimate3 -phosphate synthase (EPSPS). This enzyme participates in the biosynthesis of aromatic amino acids (phenylalanine, tyrosine and tryptophan) via the shikimate pathway in bacteria, fungi, and plants. The only enzyme known to catalyze a similar reaction in bacteria is the enzyme MurA (UDP-N-acetylglucosamine enolpyruvyl transferase, EC 2. 5.1.7), which catalyzes the first committed step in the synthesis of the peptidoglycan layer of the bacterial cell. Growth and survival of bacteria relies on the functionality of the enzyme MurA that is the target of the broadspectrum antibiotic fosfomycin. Glyphosate appears to occupy a binding site of MurA, mimicking an intermediate state of the ternary enzyme-substrates complex [6]. The similarity between the two enolpyruvyl transferases EPSPSe and MurA appears to clarify the antibacterial activity of Glyphosate. As the EPSPS-driven pathway does not exist in vertebrate cells, many scientists and environmental regulating agencies believed that glyphosate would impose minimal risks to mammals, in particular, humans [7-9]. For this reason, the shikimate pathway has been the target for the development of new anti-microbial and anti-parasite agents. In fact, glyphosate formulation has been patented as anti-parasite drug [10]. However, several emerging evidence suggested that glyphosate or GBHs (such as Roundup) can adversely affect mammalian biology via multiple mechanisms [11-13]. Downstream analyses of the functional interactions between the host and its microbiome are starting to provide mechanistic insights into these interactions. The mechanisms in which the enteric microbiome modulates specific effects on the host is not completely clear, although several mediators have been suggested as potential vehicles for such influence and might behave as effectors, enzyme cofactors and signal molecules. Such mediators include lipopolysaccharides, peptidoglycans, short-chain fatty acids, neurotransmitters and gaseous molecules $[14,15]$. Recent advances in characterizing the composition and function of individual microbial species and complex microbial communities are revealing the importance of microbial metabolism for the host immune system [16]. The gut microbiota produces an extremely diverse metabolite repertoire (such as propionic acid, a short-chain fatty acids) from the anaerobic fermentation of exogenous undigested dietary components (such as fibers) that reach the colon, as well as endogenous compounds that are generated by microorganisms and the host [17]. The single layer of epithelial cells that makes up the mucosal interface between the host and microorganisms allows microbial metabolic products to gain access to and interact with host cells, and thus influence immune responses and disease risk, in particular at high concentration [18].

GBHs have been reported to alter microbiota in soil [19], plants [20] and animals [21, 22]. A number of studies have suggested that GBHs could act as antibiotics in the mammalian gut microbiome. Recent studies have raised concerns about the health effects of glyphosate on gut microbiota of farm animal when fed feed containing residues of glyphosate. For example, farm animal studies linked epidemics of C. Botulinum-mediated diseases in dairy cows [23] to glyphosate exposure. It has been proposed that glyphosate has a potential inhibiting effect on growth of commensal bacteria, normally occupying the gut of farm animals. For example, a reduction of such beneficial bacteria could be a predisposing factor for Campylobacteriosis (campylobacter infection) described as an emerging food-borne disease [24]. Poultry is a major reservoir and source of transmission of campylobacteriosis to humans [22]. Furthermore, GBHs were also found to be capable of inducing multiple-antibiotic resistance phenotype in potential pathogens [25]. Therefore, GBHs may have the potential to modify the animal and human microbiota, which, in turn, could influence human health. However, up to date, no direct evidence has been reported to suggest any interplay between GBHs exposure and the microbiome in humans, especially during early development or in animal models exposed to GBH with low dosage relevant to humans. As denoted in the Developmental Origins of Health and Disease (DOHaD) paradigm [26], early environmental exposures are important to human health. In particular, the prenatal and neonatal period represent a narrow but 
critical window of susceptibility to myriad environmental exposures and conditions with potentially lifelong impacts on health and disease. A number of human and animal studies [27-29] associate several diseases with early-life imbalances of the gut microbiota, but it was recently pointed out the need for further evidence that GBHs, in particular at environmentally relevant doses, can result in disturbances in the gut microbiome of human and animal populations with negative health implications [30]. Furthermore, exploring the effects of GBHs on the microbiota from early-life until adulthood in different windows of susceptibility, may give a more accurate portrayal of the microbial conditions that are involved in pathogenesis. Possible alterations of the mammalian gut microbiota and its metabolites by environmental concentrations of GBHs in early development, starting from in utero, have never been explored in a controlled laboratory animal study. The present pilot study examines whether exposure to GBHs at doses of glyphosate considered to be "safe", the US ADI of 1. $75 \mathrm{mg} / \mathrm{kg}$ bw/day, defined as the chronic Reference Dose (cRfD) determined by the US EPA [31], affect the composition and diversity of the gut microbiome at early developmental stages in Sprague-Dawley rats.

\section{Methods}

\section{Experimental model}

The entire animal experiment was performed following the rules by the Italian law regulating the use and treatment of animals for scientific purposes (Legislative Decree No. 26, 2014. Implementation of the directive n. $2010 / 63 /$ EU on the protection of animals used for scientific purposes. - G.U. General Series, n. 61 of March 14th 2014). All animal study procedures were performed at the Cesare Maltoni Cancer Research Centre/ Ramazzini Institute (CMCRC/RI) (Bentivoglio, Italy). The study protocol was approved by the Ethical Committee of Ramazzini Institute. The protocol of the experiment was also approved and formally authorized by the ad hoc commission of the Italian Ministry of Health (ministerial approval n. 710/2015-PR). The CMCRC/RI animal breeding facility was the supplier for the Sprague-Dawley (SD) rats. Female breeders SD rats were placed individually in Polycarbonate cage (42x26x18cm; Tecniplast Buguggiate, Varese, Italy) with a single unrelated male until evidence of copulation was observed. After mating, matched females were housed separately during gestation and delivery. Newborns were housed with their mothers until weaning. Weaned offspring were co-housed, by sex and treatment group, not more than 3 per each cage. Cages were identified by a card indicating: study protocol code, experimental and pedigree numbers, dosage group. A shallow layer of white fir wood shavings served as bedding (supplier: Giuseppe Bordignon, Treviso, Italy). Analysis of chemical characteristics $(\mathrm{pH}$, ashes, dry weight, specific weight) and possible contamination (metals, aflatoxin, polychlorobiphenyls, organophosphorus and organochlorine pesticides) of the bedding was performed by CONSULAB Laboratories (Treviso, Italy). The cages were placed on racks, inside a single room prepared for the experiment at $22{ }^{\circ} \mathrm{C} \pm 3{ }^{\circ} \mathrm{C}$ temperature and $50 \pm 20 \%$ relative humidity. Daily checks on temperature and humidity were performed. The light was artificial and a light/dark cycle of $12 \mathrm{~h}$ was maintained. Husbandry factors stress-related were controlled: rats were kept together (same room, same rack, no more than 3 per cage) and we did not relocate cages. Noise and handling time were minimized [32].

\section{Experimental protocol}

Two groups of SD rat dams and relative pups were treated with either glyphosate or Roundup diluted in drinking water at the glyphosate concentration of 1 . $75 \mathrm{mg} / \mathrm{kg}$ bw/day. There were in total 24 F0 dams, entire litter at postnatal day (PND) 7 and PND 14, 108 F1 offspring at PND 31 and PND 57 and 60 F1 at PND 125 in this study. The F0 female breeders received the treatment through drinking water from gestation day (GD) 6 to the end of lactation. During pregnancy and lactation, embryos and offspring (F1) were all retained in the litter and might receive the test compounds mainly through their dams (F0). After weaning on PND 28 offspring were randomly distributed in two cohorts: animals belonging to the 6-week cohort were sacrificed at PND 73 \pm 2 , i.e. 6 weeks after weaning, animals belonging to the 13-week cohort were sacrificed at PND $125 \pm 2$, i.e. 13 weeks after weaning. The F1 offspring might receive the treatment from their dams starting from in utero and mainly through milk during lactation. After weaning, the offspring (F1) were treated through drinking water until sacrifice.

The timeline of the experimental animal treatment and fecal sample collection is shown in Fig. 1. As illustrated, rat fecal samples were individually collected from all animals of the F0 generation ( 8 dams) from each group before mating, at GD 5 (before the starting of the treatment), GD 13, lactation day (LD) 7 and LD 14. Fecal samples were also collected from 108 F1 pups, 18 males and 18 females from each group during lactation at PND 7 and PND 14 (corresponding to LD 7 and 14 for dams), before the achievement of puberty at PND 31, after puberty at PND 57 and in adulthood at PND 125 . Due to technical difficulty to identify fecal samples from individual pups during lactation, only pooled samples at PND 7 and PND 14 were collected for each cage from the whole litter, not distinguished by gender. After weaning, fecal samples from each pup were individually 


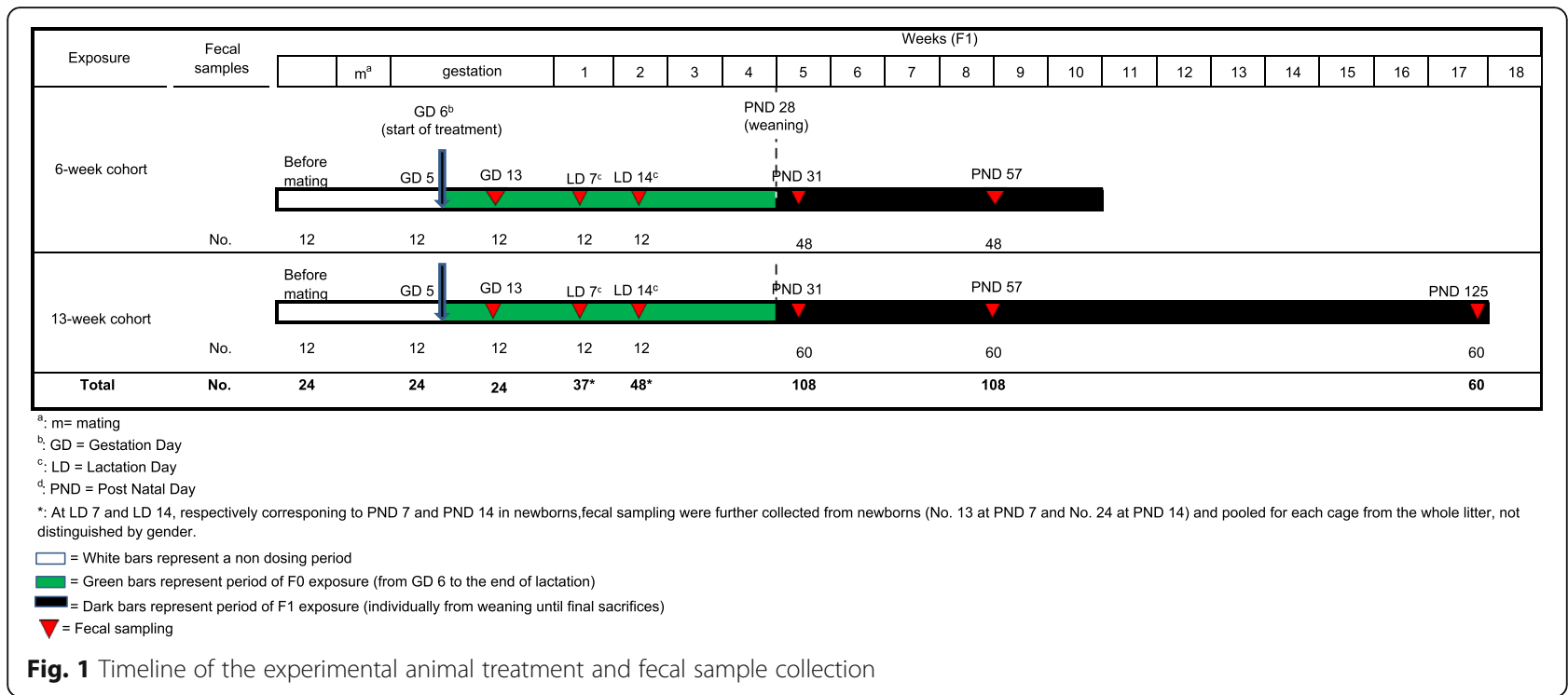

collected. About 2-3 droppings, collected directly from the anus of each animal, were preserved in cryovials on an ice bed then stored at $-20{ }^{\circ} \mathrm{C}$ until shipment on dry ice to the Icahn School of Medicine at Mount Sinai. Forceps used for collecting droppings were washed and cleaned using sterile water and $1 \%$ sodium bicarbonate between each sampling to avoid cross contamination.

\section{Bacterial 16S PCR and sequencing}

Rat fecal DNA was extracted using the QIAamp PowerFecal DNA Kit (Qiagen, Valencia, CA) following the manufacturer's instructions. Total DNA concentration was determined by Qubit 2.0 Fluorometer (Life technologies, Norwalk, CT). The phylogenetically informative V3-V4 region of $16 \mathrm{~S}$ rRNA gene was amplified using universal primer 347F/803R [33, 34] with dual-barcoding approach previously described [35]. The integrity of the $16 \mathrm{~S}$ PCR amplicons was verified by agarose gel electrophoresis. The resulting $\sim 460$-bp sized amplicons were pooled and then sequenced with the Illumina MiSeq $2 \times 250$ paired-end sequencing platform at OCS genome technology center of New York University Langone Medical Center.

\section{$16 \mathrm{~S}$ data analysis}

The sequencing data were merged and filtered to remove the merged reads with a length of $<400 \mathrm{bp}$ or the quality score of $<$ Q30 at more than $1 \%$ of bases. Sequentially, all filtered high quality reads were split by dual-barcode and trimmed of primer regions using a self-defined bash script to integrate several sequencing processing commands from fastx [36], QIIME [37, 38], and seqtk [39]. Duplicated measurements of four sample were processed and sequenced using different barcodes to test the sequencing reproducibility. Five blank samples were also sequenced and referenced to filter the possible environmental contamination during the sample procession. The split high-quality reads were further processed by QIIME 1.9.0 [37]. We used the command pick_open_reference_otus.py with the defaulted green gene 97_otus reference sequences to cluster of $>97 \%$ similar sequencing reads as an OTU using uclust [40]. Representative sequences for each OTU were aligned using PyNAST and build the phylogenetic tree. Finally, the QIIME generated biom-formatted OTU table contains the taxonomic information and absolute counts for each identified taxon in each sample.

The diversity within each microbial community, socalled alpha-diversity, was calculated using the Shannon Index [41] as metric and represented the measure of the diversity at the family and genus level. The overall microbiome dissimilarities among all samples were accessed using the weighted UniFrac distance matrices [42]. Nonmetric multiple dimensional scaling (NMDS) were used to visualize the dissimilarities. The permutational multivariate analysis of variance PERMANOVA test [43], with the maximum number of permutations $=999$, was performed to assess the significance of the overall microbiome differences between groups by collection timepoints and treatment. The PERMANOVA procedure using the [Adonis] function of the $R$ package vegan 2.0-5 [44] partitions the distance matrix among sources of variation, fits linear models to distance matrices and uses a permutation test with pseudo- $F$ ratios to obtain the $p$ values. Using the LEfSe method [45], we further selected the microbiome features significantly associated to time of collection and treatments at various taxonomic ranks.

\section{Results}

No unexpected clinical signs or symptoms were observed in the experimental animals during the in vivo 
phase. In particular, no sign of changes in maternal behavior during lactation (nesting and nursing) were observed during the experiment. There was no clinical evidence of alterations in activity or behavior in pups. Body weight, water and feed consumption both in dams and pups were no different across the groups. Litter sizes were fully comparable among groups, with mean number of live pups: control group 13.6 (range 10-16); glyphosate group 13.3 (range 11-17); Roundup group 13.9 (range 11-16).

We extract the total DNAs from 433 SD rat fecal samples. Following the timeline illustrated in Figs. 1, 120 fecal samples were collected from 24 F0 dams in three treatment groups and at five time points (before mating, GD5, GD13, LD7 and LD14). From F1 pups, we collected 313 fecal samples, in which 13 at PND 7, 24 at PND 14, 108 each at PND 31 and PND 57, and 60 at PND 125. We observed that the fecal samples of pups at PND 7 and PND 14 showed significant low DNA yields (Additional file 1: Figure S1A). We further performed microbiome survey on 433 SD rat fecal samples, and 5 water blanks using bacterial $16 \mathrm{~S}$ sequencing on Illumina MiSeq $2 \times 250$ pair-end platform. After merging and filtering by read length $>400$ bp and the quality score $>$ Q30 at more than $99 \%$ of bases, we obtained $~ 2$ million high quality reads (the average number of reads $=4576$ per sample with standard deviation $=6567$ ). The number of reads were not significant different by exposure type (Additional file 1: Figure S1A). The taxa composition was grouped by age and the exposure types and summarized in Additional file 1: Figure S1B. We also provided the complete taxonomic OTU tables in Additional file 2.

The overall microbiome dissimilarity, defined by betadiversity, was visualized by non-parametric multidimensional scaling (NMDS) plot of all samples (Fig. 2a) , dams only (Fig. 2b) and pups only (Fig. 2c). We found that the early postnatal samples at PND 7 and PND 14 were far apart from the dams at LD 7 and LD 14 while the later postnatal samples at PND 31, PND 57 and PND 125 were clustering with the dams (Fig. 2a). The mean and variance of the within-community diversity ( $\alpha$ diversity) measured by Shannon index showed that the samples from dams possessed higher, while early postnatal samples from pups showed lower $\alpha$ diversity (Fig. 2d). Student t-test showed significantly increased $\alpha$ diversity from PND 14 to PND 31 ( $p$-value $<0.05$ for all treatment groups) but no differences between samples at same age but different treatment group.

We compared the overall microbiome changes by treatment at different age groups from pups and dams. Nonmetric multidimensional scaling (NMDS) plots visualized the overall microbiome dissimilarities by treatment at PND 31 and 57 (Fig. 3a). The PERMANOVA test was used at each age group to test the significance of the differences at overall rat gut microbiome between treatment and control. The test results ( $p$-values shown in Fig. 3b) showed that the overall microbiome was significantly altered by both Roundup and glyphosate treatment compared to controls. Similarly, we also found significant differences in microbiota between Roundup and glyphosate exposed F1 pups. We also observed that the overall microbiome was significantly different by sex at PND 125 ( $p$-value $=0.028,0.007$ and 0.013 by PERMANOVA test for Glyphosate, Roundup and control group, respectively). To adjust for the sex effect, we performed additional multivariable PERMANOVA test with both treatment and sex as predictive variables. We found that those test results were consistent (Fig. 3b). However, none of the F0 dam groups showed significant differences in overall microbiota diversity..

The linear discriminant analysis effect size (LEfSe) analysis was performed using $16 \mathrm{~S}$ sequencing data from rat fecal samples in order to select particular discriminative features of the glyphosate exposure. Consistently with the overall microbiome changes by exposure at different age groups (Fig. 3), we found several significant differential taxa features associated with exposure. In particular, at PND 31, the results showed that the microbiota of both glyphosate and Roundup exposed pups had significantly higher prevalence of Prevotella genus (Bacteroidetes phylum) and Mucispirillum genus (Deferribacteres phylum) and lower prevalence of Lactobacillus genus (Firmicutes phylum) and Aggregatibacter genus (Proteobacteria phylum) (Fig. 4a 1-2). However, some of the selected features were treatment specific. For instance, among the most significant features with LDA score $>3.0$ and pvalue $<0.05$, we found increased Blautia genus (Firmicutes phylum) and decreased Streptococcus genus (Firmicutes phylum) and Rothia genus (Actinobacteria phylum) only in glyphosate exposed PND 31 pups, but not in Roundup exposed samples. In contrast, increased Parabacteroides genus (Bacteroidetes phylum) and Veillonella genus (Firmicutes phylum) were only found in Roundup exposed pups, but not in glyphosate exposed samples at PND 31. Between two exposures (Fig. 4a 3), Roundup exposed pups showed increased Clostridia class (Firmicutes phylum), in particular, Blautia genus and Actinobacteria class (Actinobacteria phylum), in particular, Rothia and Bifidobacterium genera at PND 31. Furthermore, we found the treatment associated taxa features were not consistent at different postnatal time points. Many features selected at PND 31 did not appeared at PND 57 (Fig. 4a 4-6, Additional file 3: Figure S2), suggesting the less stability of early-life microbiota and continuous effect on gut microbiota by the exposure. When counting the total abundance $\%$ of the significant differential taxa by treatments, the pups showed much higher impact by exposure than the dams (Fig. 4b). 


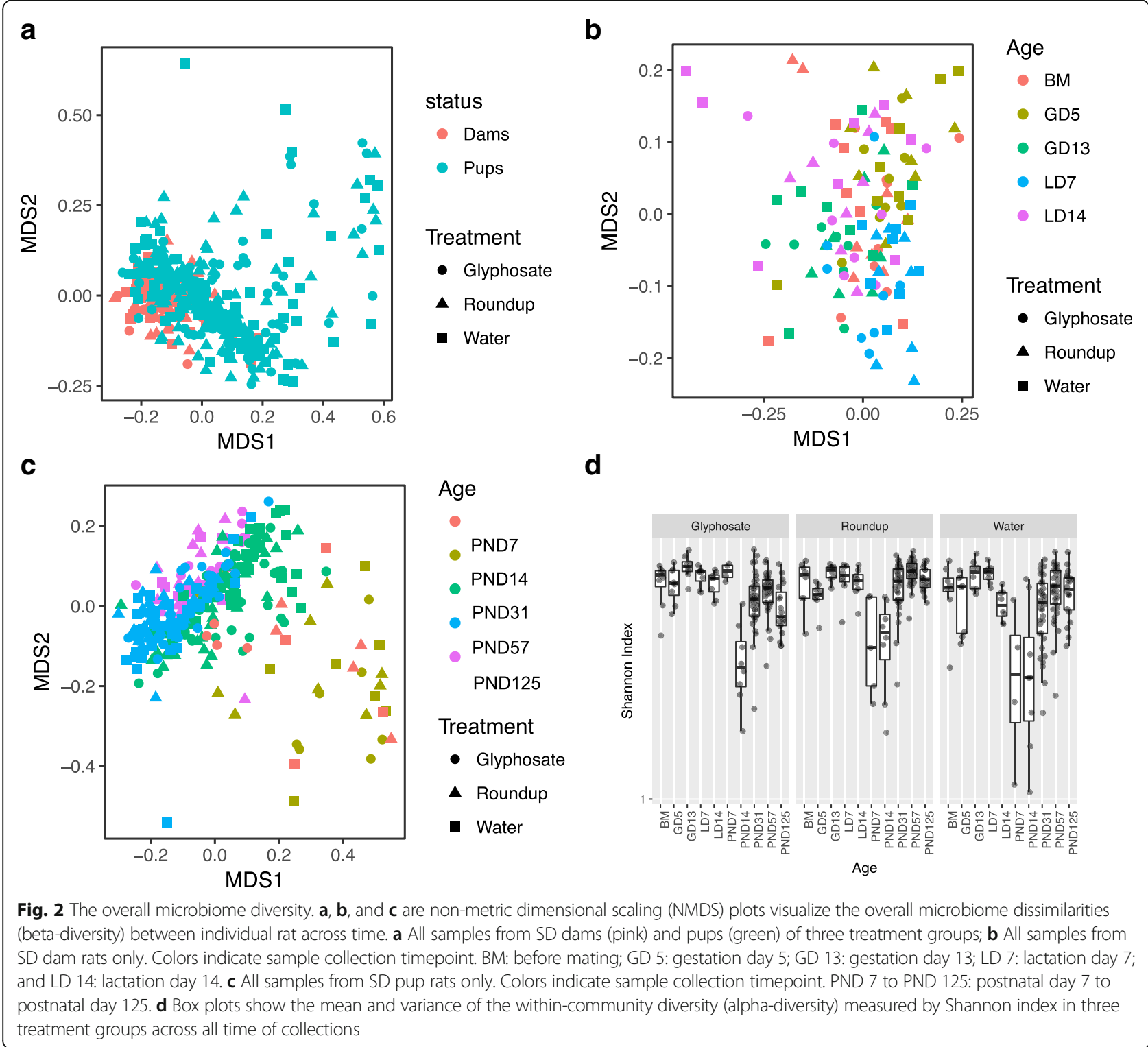

\section{Discussion}

GBHs are the most applied herbicides worldwide; humans are commonly exposed to these environmental chemicals at a wide range of doses depending upon the job setting (farming vs. food consumption) and route of exposure (ingestion vs. inhalation). Environmental contamination from GBHs is now ubiquitous and residues of glyphosate has been found in air [46], groundwater [47], drinking-water [48], crops [49], food [50] and animal feed [51]. The possible effects of GBHs on human health are the subject of an intense public debate, for both its potential carcinogenic and non-carcinogenic effects, including endocrine disruption [52, 53], neurotoxicity [54], developmental and reproductive toxicity [55], autoimmunity [56], gastrointestinal disorders [57], obesity, diabetes [58-60], and other metabolic and cardiovascular disorders [61] and central nervous system dysfunctions such as learning and memory impairment, anxiety, stress, depression [62] and autism [63]. These chronic pathologies (non-communicable diseases NCDs) may occur even at doses that are much lower than the ones considered during risk assessment, in particular during sensitive periods of life (such as fetal development) [7, 22].

Recent advances in human microbiome research suggested that the gut microbiome is a key player in human metabolism [64-66]. It is thus reasonable to hypothesize that exposure to environmental chemicals may modify the gut microbiome and its metabolites and ultimately influence human health. Microbiota-generated metabolites and their cellular and molecular components are increasingly being recognized as an essential part of 


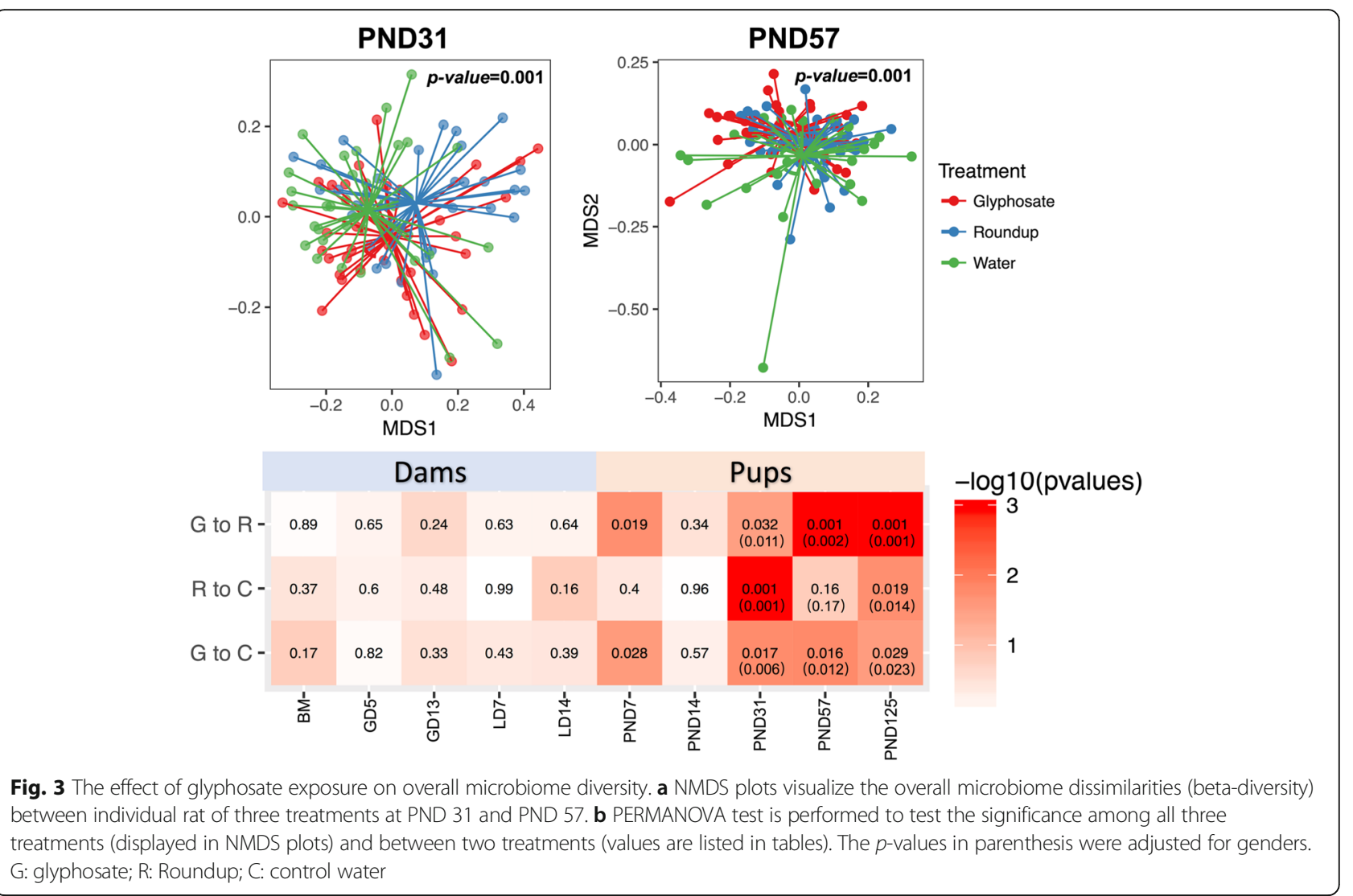

human physiology, with profound effects on the homeostasis of the host organism. Unfortunately, determining the concentrations of these biologically active substances in target cells presents serious difficulties related to the extraction and processing of samples, especially faecal material, and the limitations of currently available measurement techniques [15]. Meta-omics and evolving computational frameworks will hopefully lead to the systematic prediction and discovery of more microbial metabolites and components involved in neuroendocrine, immune, metabolic, and epigenetic pathways.

Rats are proposed to be more representative of the human gut microbiota than mice because the gut bacterial communities of humanized rats more closely reflect the gut microbiota of human donors $[67,68]$. We have previously used our animal model, SD rats, to study the effect of postnatal low-dose exposure to environmental chemicals on windows of susceptibility and on the gut microbiome. The study [69] showed the low-level phthalate, paraben and triclosan exposure altered the gut microbiome of adolescent rats. These results are consistent with other studies, indicating our animal model as a suitable model for studying microbiome [70, 71].

Since glyphosate has shown enzyme inhibition activity in plants and microorganisms, we therefore postulate that low-dose exposure to glyphosate or GBHs may also modulate the composition of the gut microbiome. In this study, when compared to the adult rat dams, the gut microbiome of pups at PND 7 and 14 showed lower taxonomical richness but higher variance within sample and higher sample-to-sample dissimilarity [69]. One pitfall of our study was that direct measurements of exposure to GBHs in milk was not performed [72]. In our pilot study we simply reproduced the human exposure, which includes lactation as only source of nourishment for pups from birth until around PND 21. The shortcomings concerning the analysis of glyphosate in breast milk are mainly related to the difficulty and stressing technical procedure for collecting milk from dams and to the complex nature of the breast milk matrix. Indeed, milk is an aqueous mixture of carbohydrates, proteins and fat. Analytical methods developed for watery matrices cannot be directly transferred to breast milk. In April 2014, a non-peer-reviewed report was published, in which glyphosate in breast milk of American mothers was detected in 3 out of 10 samples ranging from 76 to $166 \mathrm{ng} / \mathrm{mL}$. In this study, the concentration of glyphosate in milk samples was determined by enzyme-linked immunosorbent assay (ELISA) [73]. The limit of quantification (LOQ) of the assay was given as $75 \mu \mathrm{g} / \mathrm{L}$ in milk. Other studies, based on liquid chromatography-tandem mass spectrometry (LC-MS/MS) and a gas 


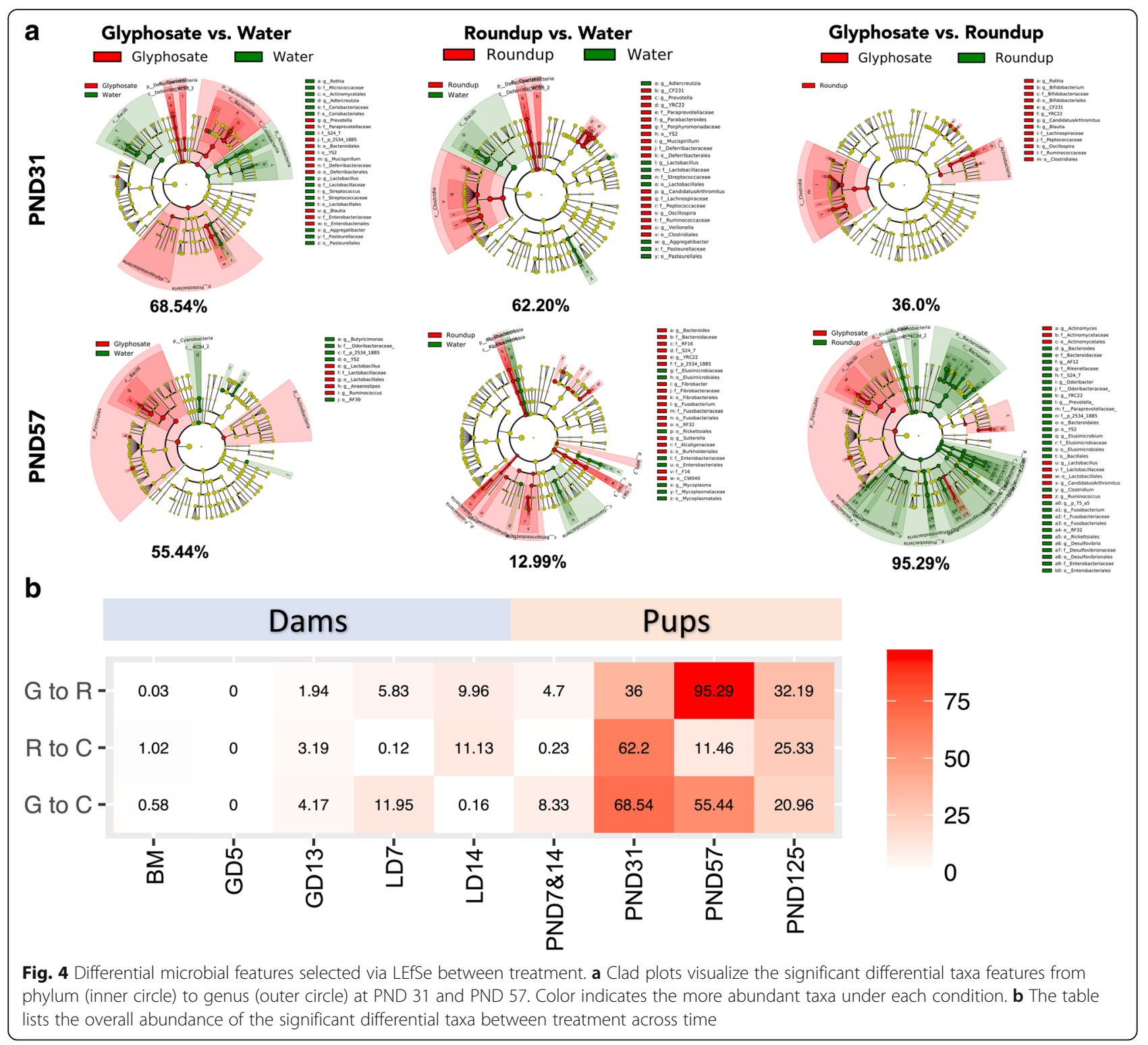

chromatography-tandem mass spectrometry (GC-MS/ MS) methods, have found no evidence of transfer of glyphosate into milk. Both methods have been fully validated and reported as suitable for the determination of glyphosate with an LOQ of $1 \mathrm{ng} / \mathrm{mL}[72,74]$. Nevertheless, future independent research is needed, considering different educational and ethnic backgrounds, location of residence (e.g., urban compared with rural), occupational and dietary glyphosate exposure and adequate sample size of the cohort.

Our results revealed that both glyphosate and glyphosate formulated Roundup, at doses admitted in humans, including children and pregnant women, significantly altered the microbiota diversity and resulted in prominent changes at multiple taxon in exposed pups. However, those effects on microbiota were not significant in the adult dams. Previous evidence has shown that the gut microbiota at postnatal age is less stable than at adult age and it changes over the first several years of life [75]. The maturation of the gut microbiota has been proven to be affected by multiple factors, for instance, diet, medications, host genetics, etc. [76]. Disruption of the microbiota during its maturation by low doses of various environmental chemicals has been showed to alter host phenotypes, such as weight, metabolism and other disease risk [77]. Our data suggests that the prepubertal age microbiota is more sensitive to GBH exposure compared to the adult microbiota, therefore the postnatal age is likely a "window of susceptibility" for GBHs to modulate the gut microbiome.

Furthermore, our results showed that the overall microbiome diversity and composition were significantly 
different between Roundup and glyphosate, suggesting possible synergistic effects of the mixed formulation on gut microbiota. As most of GBHs contains multiple surfactants and adjuvants might act differently than glyphosate alone, it is not only important to understand the individual effects of glyphosate, but also the synergistic impact of mixed formulations. In fact adjuvants might act alone or in a synergistic manner and increase the toxic effects of glyphosate [78-81].

In addition, both clinical and experimental studies showed impact of gut microbiota on the gut-brain axis (which mainly includes the immune, neuroendocrine, and neural pathways) [82-84] in an age-dependent manner [85]. Gut bacteria communicating with the host through the microbiota-gut-brain axis could influence brain and behavior [86]. In particular, the changes at postnatal microbiota may affect the neurvous system, reflecting by changes in levels of pituitary hormones including ACTH [83, 87], cortisol, BNDF [88] and etc. Sprague-Dawley rats represent an excellent animal model to explore these early-life effects as their microbiome is more similar to that of humans than the microbiota profile of mice [67].

This study has some limitations. First, the actual levels of GBHs that reached the fetus during gestation or through milk consumption postnatally by the offspring cannot be accurately estimated. Second, we only collected maternal feces so that we cannot fully evaluate the role of maternal microbiota in the fetal development without the maternal sample/data collection from oral, vaginal and other body sites. Indeed, in recent years it is becoming apparent that, besides breast milk, other sources could allow maternal-offspring microbial transfer. Rodents "inherit" their microbiomes in a similar fashion to all placental mammals, including humans: through vaginal delivery and close maternal association throughout the neonatal period (vertical transmission). Maternal vaginal, skin, mammary fecal and oral microbiomes, microbial spreading in bedding are efficiently transmitted to offspring and represent other possible mechanisms of maternal influences on pups intestinal colonization [89]. Finally, the microbiome survey used a cost-effective $16 \mathrm{~S}$ amplicon targeted sequencing approach. This technique allows us to identify differential taxa compositions by exposure only to genus level. Additional meta-genomics and meta-transcriptomic analysis may need to visualize the functional and metabolic alternations and identify bacterial features at species/strain level. In addition, given the differences in taxonomic composition of the microbiomes of rats and humans, the extent to which the results of this analysis can be relevant to humans is not clear. Future work should investigate how the route and concentration of exposure impact the rat microbiome, and quantify how these perturbations may impact subsequent health outcomes. Nevertheless, these data strongly indicate that GBHs exposure can exerts biological effects early in development which may have long-lasting health effects later in life.

\section{Conclusion}

Our pilot study provides initial evidence that maternal exposure to commonly used GBHs, at doses currently considered as acceptable in humans, is capable of modifying the gut microbiota in rat pups, in particular before puberty (PND 31). Further long-term investigations are necessary to elucidate if the shift in the microbiota induced by GBHs exposure is contributing to the downstream other health effects. Nevertheless, understanding the microbiota changes during this critical window of susceptibility could be of great importance for disease prevention. The potential health effects of GBHs during development, such as childhood, warrant further investigation.

\section{Additional files}

\begin{abstract}
Additional file 1: Figure S1. 16 S microbiome profiling. A. Dot plot shows the distribution of the number of reads in three treatment groups. The Wilcoxon test significance between two groups was listed in table on the right and the diagonal of the table shows the average reads of each group. B. Box plot shows the mean and variation of total DNA concentrations from rat fecal samples. $\mathbf{C}$. Bar plot showed the mean abundance of microbial composition at phylum level for each treatment and time of collection. (PDF $174 \mathrm{~kb}$ )
\end{abstract}

Additional file 2: $16 \mathrm{~S}$ OTU table in biom format. (BIOM $6871 \mathrm{~kb}$ )

Additional file 3: Figure S2. The changes of lactobacillus and Prevotella during the time of sampling. Line plots show the mean and standard error of relative abundance\% of Lactobacillus (upper figure) and Prevotella (lower figure) during the time of sampling from PND 7 to PND 125. (PDF $543 \mathrm{~kb}$ )

\section{Abbreviations}

ACTH: Adrenocorticotropic hormone; AMPA: Aminomethylphosphonic acid; BDNF: brain-derived neurotrophic factor; CMCRC: Cesare Maltoni Cancer Research Center; DOHaD: Developmental origins of health and disease; ELISA: Enzyme-linked immunosorbent assay; EPSPS: 5-Enolpyruvylshikimate-3phosphate synthase; EU: European Union; GBH: Glyphosate-based herbicides; GC-MS/MS: gas chromatography-tandem mass spectrometry;

GD: Gestational day; GM: Genetically modified; LC-MS/MS: Chromatographytandem mass spectrometry; LD: Lactating day; LDA: Linear discriminant analysis; LEfSe: Linear discriminant analysis effect size; LOQ: Limit of quantification; NCDs: Non-communicable diseases; NMDS: Non-metric multiple dimensional scaling; OUT: operational taxonomic unit; PND: Post natal day; PyNAST: Python nearest alignment space termination; QIIME: Quantitative insights into microbial ecology; RI: Ramazzini Institute; SD: Sprague-Dawley; US ADI: United States Acceptable Daily Intake

\section{Acknowledgements}

We thank the over 30.000 associates and volunteers of the Ramazzini Institute that made this pilot study possible through their commitment and generosity. We thank the Municipality of Bologna, the Emilia-Romagna Region, and the International Society of Doctors for Environment for organizing several events to promote this pilot study; "Coop Reno" and "Coopfond Fondo Mutualistico Legacoop" for supporting our research activity. We thank the OCS genome technology center of New York University Langone Medical Center for the library preparation and sequencing service. 


\section{Funding}

This work was funded by Institution fund of Ramazzini Institute, Bologna, Italy; Fondazione del Monte di Bologna e Ravenna (Bank Foundation), Bologna, Italy; MSSM seed fund $(\mathrm{JH})$ and $\mathrm{CL}$ and $\mathrm{JC}$ were supported by the $\mathrm{NIH} / \mathrm{NIEHS}$ P30ES023515.

\section{Availability of data and materials}

165 rRNA gene sequencing information has been deposited into EMBL

Nucleotide Sequence Database (ENA) with Project ID PRJEB24653 (ERP106496).

\section{Authors' contributions}

QXM performed the fecal sample processing, PCR and library preparation, performed microbial sequencing analysis and bioinformatics, and drafted the manuscript. FM, SP, DM participated in the design of the study, performed the animal experiments and sample collection, and drafted the manuscript. $I M, A V, L B$ and LF performed the animal experiments and collected the samples. CL helped to draft the manuscript. FB and JC supervised the study, participated in the design of the study and helped to draft the manuscript. $\mathrm{JH}$ conceived of the overall study, supervised the overall experiment, implemented the bioinformatics, and coordination and draft the manuscript. All authors read and approved the final manuscript.

\section{Ethics approval and consent to participate}

Not applicable.

\section{Competing interests}

The authors declare that they have no competing interests.

\section{Publisher's Note}

Springer Nature remains neutral with regard to jurisdictional claims in published maps and institutional affiliations.

\section{Author details}

${ }^{1}$ Department of Genetics and Genomic Sciences, Icahn School of Medicine at Mount Sinai, 1428 Madison, New York, NY 10029, USA. ${ }^{2}$ Department of Thoracic Surgery, Jiangsu Key Laboratory of Molecular and Translational Cancer Research, Cancer Institute of Jiangsu Province, Nanjing Medical University Affiliated Cancer Hospital, Nanjing, China. ${ }^{3}$ Cesare Maltoni Cancer Research Center (CMCRC), Ramazzini Institute (RI), Via Saliceto, 3, 40010 Bentivoglio, Bologna, Italy. ${ }^{4}$ Department of Veterinary Medical Sciences, University of Bologna, Bologna, Italy. ${ }^{5}$ Department of Agricultural Sciences, University of Bologna, Bologna, Italy. ${ }^{6}$ Department of Environmental Medicine and Public Health, Icahn School of Medicine at Mount Sinai, 1428 Madison, New York, NY 10029, USA.

\section{Received: 2 February 2018 Accepted: 10 May 2018}

\section{Published online: 29 May 2018}

\section{References}

1. Franz J, Mao M. Sikorski J. A Unique Global Herbicide: Glyphosate; 1997.

2. Benbrook CM. Trends in glyphosate herbicide use in the United States and globally. Environ Sci Eur. 2016;28:3.

3. Myers JP, Antoniou MN, Blumberg B, Carroll L, Colborn T, Everett LG, et al. Concerns over use of glyphosate-based herbicides and risks associated with exposures: a consensus statement. Environ Health. 2016;15:19.

4. Bovey RW, Miller FR, Baur JR. Preharvest desiccation of grain Sorghum with glyphosate. Agron J. 1975;67:618-21.

5. Toledo MZ, Ishizuka MS, Cavariani C, De Barros F-NJ, Picoli LB. Pre-harvest desiccation with glyphosate and quality of stored soybean seeds. Semin Agrar. 2014;35:765-73.

6. Schönbrunn E, Eschenburg S, Shuttleworth WA, Schloss JV, Amrhein N, Evans $J \mathrm{~N}$, et al. Interaction of the herbicide glyphosate with its target enzyme 5-enolpyruvylshikimate 3-phosphate synthase in atomic detail. Proc Natl Acad Sci U S A. 2001;98:1376-80.

7. On C, Peer P. Conclusion on the peer review of the pesticide risk assessment of the active substance glyphosate. EFSA J. 2015;13:4302.

8. ECHA. Echa's committee for risk assessment (rac). 2017.

9. EPA. Draft human health and ecological risk assessments for glyphosate. 2017.

10. Abraham W. Glyphosate formulations and their use for the inhibition of 5enolpyruvylshikimate-3-phosphate synthase. 2010. US Patent $n$. US7771736B2
11. Tarazona JV, Court-Marques D, Tiramani M, Reich H, Pfeil R, Istace F, et al. Glyphosate toxicity and carcinogenicity: a review of the scientific basis of the European Union assessment and its differences with IARC. Arch Toxicol. 2017;91:2723-43.

12. Samsel A, Seneff S. Glyphosate, pathways to modern diseases III: manganese, neurological diseases, and associated pathologies. Surg Neurol Int. 2015;6:45

13. IARC. Some organophosphate insecticides and herbicides. 2015.

14. Frye RE, Nankova B, Bhattacharyya S, Rose S, Bennuri SC, MacFabe DF. Modulation of immunological pathways in autistic and neurotypical lymphoblastoid cell lines by the enteric microbiome metabolite propionic acid. Front Immunol. 2017;8:1670.

15. Oleskin AV, Shenderov BA. Neuromodulatory effects and targets of the SCFAs and gasotransmitters produced by the human symbiotic microbiota. Microb Ecol Heal Dis. 2016;27:30971.

16. MacFabe DF. Enteric short-chain fatty acids: microbial messengers of metabolism, mitochondria, and mind: implications in autism spectrum disorders. Microb Ecol Heal Dis. 2015;26:28177.

17. den Besten G, van Eunen K, Groen AK, Venema K, Reijngoud D-J, Bakker BM. The role of short-chain fatty acids in the interplay between diet, gut microbiota, and host energy metabolism. J Lipid Res. 2013;54:2325-40.

18. Rooks MG, Garrett WS. Gut microbiota, metabolites and host immunity. Nat Rev Immunol. 2016;16:341-52

19. Newman MM, Hoilett N, Lorenz N, Dick RP, Liles MR, Ramsier C, et al. Glyphosate effects on soil rhizosphere-associated bacterial communities. Sci Total Environ. 2016;543:155-60.

20. Duke SO, Lydon J, Koskinen WC, Moorman TB, Chaney RL, Hammerschmidt R. Glyphosate effects on plant mineral nutrition, crop rhizosphere microbiota, and plant disease in glyphosate-resistant crops. J Agric Food Chem. 2012;60:10375-97.

21. Krüger M, Shehata AA, Schrödl W, Rodloff A. Glyphosate suppresses the antagonistic effect of enterococcus spp. on Clostridium botulinum. Anaerobe. 2013;20:74-8.

22. Shehata AA, Schrödl W, Aldin AA, Hafez HM, Krüger M. The effect of glyphosate on potential pathogens and beneficial members of poultry microbiota in vitro. Curr Microbiol. 2013;66:350-8.

23. Ackermann W, Coenen M, Schrödl W, Shehata AA, Krüger M. The influence of glyphosate on the microbiota and production of botulinum neurotoxin during ruminal fermentation. Curr Microbiol. 2014;70:374-82.

24. Houf K, Stephan R. Isolation and characterization of the emerging foodborn pathogen Arcobacter from human stool. J Microbiol Methods. 2007;68:408-13.

25. Kurenbach B, Marjoshi D, Amábile-Cuevas CF, Ferguson GC, Godsoe W, Gibson $P$, et al. Sublethal exposure to commercial formulations of the herbicides dicamba, 2,4-dichlorophenoxyacetic acid, and glyphosate cause changes in antibiotic susceptibility in Escherichia coli and Salmonella enterica serovar typhimurium. MBio. 2015;6(2):e00009-1.

26. Heindel JJ, Vandenberg LN. Developmental origins of health and disease: a paradigm for understanding disease cause and prevention. Curr Opin Pediatr. 2015;27:248-53.

27. Wang Z, Klipfell E, Bennett BJ, Koeth R, Levison BS, Dugar B, et al. Gut flora metabolism of phosphatidylcholine promotes cardiovascular disease. Nature. 2011;472:57-65.

28. Adams JB, Johansen LJ, Powell LD, Quig D, Rubin RA. Gastrointestinal flora and gastrointestinal status in children with autism - comparisons to typical children and correlation with autism severity. BMC Gastroenterol. 2011;11:22

29. Turnbaugh PJ, Ley RE, Mahowald MA, Magrini V, Mardis ER, Gordon JI. An obesity-associated gut microbiome with increased capacity for energy harvest. Nature. 2006;444:1027-31.

30. Mesnage R, Antoniou MN. Facts and fallacies in the debate on glyphosate toxicity. Front Public Heal. 2017;5:316.

31. EPA. Reregistration eligibility decision (RED) glyphosate. 1993.

32. Laukens D, Brinkman BM, Raes J, De Vos M, Vandenabeele P. Heterogeneity of the gut microbiome in mice: guidelines for optimizing experimental design. FEMS Microbiol Rev. 2015;40:117-32.

33. Franzén O, Hu J, Bao X, Itzkowitz SH, Peter I, Bashir A. Improved OTUpicking using long-read 165 rRNA gene amplicon sequencing and generic hierarchical clustering. Microbiome. 2015;3:43.

34. Nossa CW, Oberdorf WE, Yang L, Aas JA, Paster BJ, Desantis TZ, et al. Design of 165 rRNA gene primers for 454 pyrosequencing of the human foregut microbiome. World J Gastroenterol. 2010;16:4135-44. 
35. Torres J, Bao X, Goel A, Colombel JF, Pekow J, Jabri B, et al. The features of mucosa-associated microbiota in primary sclerosing cholangitis. Aliment Pharmacol Ther. 2016;43:790-801.

36. Schmieder R, Edwards R. Quality control and preprocessing of metagenomic datasets. Bioinformatics. 2011;27:863-4.

37. Caporaso JG, Kuczynski J, Stombaugh J, Bittinger K, Bushman FD, Costello EK, et al. QIIME allows analysis of high-throughput community sequencing data. Nat Methods. 2010;7:335-6.

38. Caporaso JG, Kuczynski J, Stombaugh J, Bittinger K, Bushman FD, Costello EK, et al. Correspondence QIIME allows analysis of high- throughput community sequencing data intensity normalization improves color calling in SOLiD sequencing. Nat Publ Gr. 2010;7:335-6.

39. Shen W, Le S, Li Y, Hu F. SeqKit: a cross-platform and ultrafast toolkit for FASTA/Q file manipulation. PLoS One. 2016;11(10):e0163962.

40. Edgar RC. Search and clustering orders of magnitude faster than BLAST. Bioinformatics. 2010;26:2460-1.

41. Shannon CE. A mathematical theory of communication. Bell Syst Tech J. July 1928;1948(27):379-423.

42. Lozupone C, Knight R. UniFrac: a new phylogenetic method for comparing microbial communities. Appl Environ Microbiol. 2005;71:8228-35.

43. Anderson MJ. A new method for non parametric multivariate analysis of variance. Austral Ecol. 2001;26:32-46.

44. Oksanen J. Multivariate analysis of ecological communities in R: vegan tutorial. R Doc. June 10, 2015. http://www.cc.oulu.fi/ jarioksa/opetus/ metodi/vegantutor.pdf. Accessed 14 May 2018.

45. Segata N, Izard J, Waldron L, Gevers D, Miropolsky L, Garrett WS, et al. Metagenomic biomarker discovery and explanation. Genome Biol. 2011;12:R60.

46. Majewski MS, Coupe RH, Foreman WT, Capel PD. Pesticides in Mississippi air and rain: a comparison between 1995 and 2007. Environ Toxicol Chem. 2014;33:1283-93.

47. Battaglin WA, Meyer MT, Kuivila KM, Dietze JE. Glyphosate and its degradation product AMPA occur frequently and widely in U.S. soils, surface water, groundwater, and precipitation. J Am Water Resour Assoc. 2014;50: 275-90.

48. Rendón-Von Osten J, Dzul-Caamal R. Glyphosate residues in groundwater, drinking water and urine of subsistence farmers from intensive agriculture localities: a survey in Hopelchén, Campeche, Mexico. Int J Environ Res Public Health. 2017;14(6):E595.

49. Cuhra M. Review of GMO safety assessment studies: glyphosate residues in roundup ready crops is an ignored issue. Environ Sci Eur. 2015;27:20.

50. EFSA. The 2014 European Union report on pesticide residues in food. EFSA J. 2016;14:4611.

51. Mesnage R, Defarge N, Rocque LM, De Vendômois JS, Séralini GE. Laboratory rodent diets contain toxic levels of environmental contaminants: implications for regulatory tests. PLoS One. 2015;10(7):e0128429.

52. Gasnier C, Dumont C, Benachour N, Clair E, Chagnon MC, Séralini GE. Glyphosate-based herbicides are toxic and endocrine disruptors in human cell lines. Toxicology. 2009;262:184-91.

53. Romano MA, Wisniewski P, Viau P, Romano RM, Campos DA, Bernardi MM, et al. Glyphosate impairs male offspring reproductive development by disrupting gonadotropin expression. Arch Toxicol. 2012;86:663-73.

54. Cattani D, de Liz Oliveira Cavalli VL, Heinz Rieg CE, Domingues JT, Dal-Cim $\mathrm{T}$, Tasca $\mathrm{Cl}$, et al. Mechanisms underlying the neurotoxicity induced by glyphosate-based herbicide in immature rat hippocampus: involvement of glutamate excitotoxicity. Toxicology. 2014;320:34-45.

55. Williams AL, Watson RE, Desesso JM. Developmental and reproductive outcomes in humans and animals after glyphosate exposure: a critical analysis. Journal of Toxicology and Environmental Health-Part B: Critical Reviews. 2012;15:39-96.

56. Chen J, Chia N, Kalari KR, Yao JZ, Novotna M, Soldan MMP, et al. Multiple sclerosis patients have a distinct gut microbiota compared to healthy controls. Sci Rep. 2016;6:28484

57. Cenit MC, Olivares M, Codoñer-Franch P, Sanz Y. Intestinal microbiota and celiac disease: Cause, consequence or co-evolution? Nutrients. 2015;7:6900-23.

58. Cani PD, Geurts L, Matamoros S, Plovier H, Duparc T. Glucose metabolism: focus on gut microbiota, the endocannabinoid system and beyond. Diabetes and Metabolism. 2014:40:246-57.

59. De Goffau MC, Luopajärvi K, Knip M, Ilonen J, Ruohtula T, Härkönen T, et al. Fecal microbiota composition differs between children with $\beta$-cell autoimmunity and those without. Diabetes. 2013;62:1238-44.
60. Knip $M$, Siljander $H$. The role of the intestinal microbiota in type 1 diabetes mellitus. Nat Rev Endocrinol. 2016;12:154-67.

61. Tang WHW, Hazen SL. The contributory role of gut microbiota in cardiovascular disease. J Clin Invest. 2014;124:4204-11.

62. Dinan TG, Cryan JF. Melancholic microbes: a link between gut microbiota and depression? Neurogastroenterol Motil. 2013:25:713-9.

63. Vuong HE, Hsiao EY. Emerging roles for the gut microbiome in autism Spectrum disorder. Biol Psychiatry. 2017;81(5):411-23.

64. Turnbaugh PJ, Hamady M, Yatsunenko T, Cantarel BL, Duncan A, Ley RE, et al. A core gut microbiome in obese and lean twins. Nature. 2009;457:480-4.

65. Turnbaugh PJ, Gordon J. The core gut microbiome, energy balance and obesity. J Physiol. 2009;587(Pt 17):4153-8.

66. Cho I, Blaser MJ. The human microbiome: at the interface of health and disease. Nat Rev Genet. 2012;13:260-70.

67. Flemer B, Gaci N, Borrel G, Sanderson IR, Chaudhary PP, Tottey W, et al. Fecal microbiota variation across the lifespan of the healthy laboratory rat. Gut Microbes. 2017:8:428-39.

68. Wos-Oxley ML, Bleich A, Oxley APA, Kahl S, Janus LM, Smoczek A, et al. Comparative evaluation of establishing a human gut microbial community within rodent models. Gut Microbes. 2012;3:234-49.

69. Hu J, Raikhel V, Gopalakrishnan K, Fernandez-Hernandez H, Lambertini L, Manservisi $F$, et al. Effect of postnatal low-dose exposure to environmental chemicals on the gut microbiome in a rodent model. Microbiome. 2016; $4(1): 26$.

70. Gao B, Tu P, Bian X, Chi L, Ru H, Lu K. Profound perturbation induced by triclosan exposure in mouse gut microbiome: a less resilient microbial community with elevated antibiotic and metal resistomes. BMC Pharmacol Toxicol. 2017;18(1):46.

71. Yee AL, Gilbert JA. Is triclosan harming your microbiome? Science. 2016;353: 348-9.

72. Mcguire MK, Mcguire MA, Price WJ, Shafii B, Carrothers JM, Lackey KA, et al. Glyphosate and aminomethylphosphonic acid are not detectable in human milk. Am J Clin Nutr. 2016;103:1285-90.

73. Honeycutt Z, Rowlands H. Glyphosate Testing Full Report: Findings in American Mothers' Breast Milk, Urine and Water. 2014. https://www. momsacrossamerica.com/glyphosate_testing_results. Accessed 14 May 2018

74. Steinborn A, Alder L, Michalski B, Zomer P, Bendig P, Martinez SA, et al. Determination of glyphosate levels in breast milk samples from Germany by LC-MS/MS and GC-MS/MS. J Agric Food Chem. 2016;64:1414-21.

75. Rodríguez JM, Murphy K, Stanton C, Ross RP, Kober Ol, Juge N, et al. The composition of the gut microbiota throughout life, with an emphasis on early life. Microb Ecol Heal Dis. 2015;26:26050.

76. Jakobsson HE, Jernberg C, Andersson AF, Sjölund-Karlsson M, Jansson JK, Engstrand L. Short-term antibiotic treatment has differing long-term impacts on the human throat and gut microbiome. PLoS One. 2010;5:e9836.

77. Suez J, Korem T, Zeevi D, Zilberman-Schapira G, Thaiss CA, Maza O, et al. Artificial sweeteners induce glucose intolerance by altering the gut microbiota. Nature. 2014;514:181-6.

78. Coalova I. Ríos de Molina M del C, Chaufan G. Influence of the spray adjuvant on the toxicity effects of a glyphosate formulation. Toxicol Vitr. 2014;28:1306-11.

79. Mesnage R, Bernay B, Séralini GE. Ethoxylated adjuvants of glyphosatebased herbicides are active principles of human cell toxicity. Toxicology. 2013:314:122-8.

80. Defarge N, Takács E, Lozano VL, Mesnage R, de Vendômois JS, Séralini GE, et al. Co-formulants in glyphosate-based herbicides disrupt aromatase activity in human cells below toxic levels. Int J Environ Res Public Health. 2016; 13(3):E264.

81. Williams GM, Kroes R, Munro IC. Safety evaluation and risk assessment of the herbicide roundup and its active ingredient, glyphosate, for humans. Regul Toxicol Pharmacol. 2000;31(2 I):117-65.

82. Foster JA, McVey Neufeld KA. Gut-brain axis: how the microbiome influences anxiety and depression. Trends Neurosci. 2013;36:305-12.

83. Heijtz RD, Wang S, Anuar F, Qian Y, Bjorkholm B, Samuelsson A, et al. Normal gut microbiota modulates brain development and behavior. Proc Natl Acad Sci. 2011;108:3047-52.

84. Koloski NA, Jones M, Kalantar J, Weltman M, Zaguirre J, Talley NJ. The braingut pathway in functional gastrointestinal disorders is bidirectional: a 12year prospective population-based study. Gut. 2012;61:1284-90. 
85. Carabotti M, Scirocco A, Maselli MA, Severi C. The gut-brain axis: interactions between enteric microbiota, central and enteric nervous systems. Ann Gastroenterol. 2015;28:203-9.

86. Liang S, Wang T, Hu X, Luo J, Li W, Wu X, et al. Administration of Lactobacillus helveticus NS8 improves behavioral, cognitive, and biochemical aberrations caused by chronic restraint stress. Neuroscience. 2015;310:561-77.

87. Sudo N, Chida Y, Aiba Y, Sonoda J, Oyama N, Yu XN, et al. Postnatal microbial colonization programs the hypothalamic-pituitary-adrenal system for stress response in mice. J Physiol. 2004;558:263-75.

88. Bercik P, Denou E, Collins J, Jackson W, Lu J, Jury J, et al. The intestinal microbiota affect central levels of brain-derived neurotropic factor and behavior in mice. Gastroenterology. 2011;141(2):599-609.

89. Nguyen TLA, Vieira-Silva S, Liston A, Raes J. How informative is the mouse for human gut microbiota research? Dis Model Mech. 2015;8:1-16.

Ready to submit your research? Choose BMC and benefit from:

- fast, convenient online submission

- thorough peer review by experienced researchers in your field

- rapid publication on acceptance

- support for research data, including large and complex data types

- gold Open Access which fosters wider collaboration and increased citations

- maximum visibility for your research: over $100 \mathrm{M}$ website views per year 